\title{
National outbreak of Salmonella Enteritidis phage type 14b in England, September to December 2009: case- control study
}

K Janmohamed (Kulsum@doctors.org.uk) ${ }^{1}$, D Zenner ${ }^{1,2}$, C Little $^{1}$, C Lane $^{1}$, J Wain ${ }^{1}$, A Charlett $^{1}$, B Adak ${ }^{1}$, D Morgan $^{1}$

1. Health Protection Agency, Centre for Infections, London, United Kingdom

2. London School of Hygiene and Tropical Medicine, London, United Kingdom

Citation style for this article:

Janmohamed K, Zenner D, Little C, Lane C, Wain J, Charlett A, Adak B, Morgan D. National outbreak of Salmonella Enteritidis phage type 14b in England, September to December 2009: case-control study. Euro Surveill. 2011;16(15):pii=19840. Available online: http://www.eurosurveillance.org/ViewArticle.aspx?Articleld=19840

Article published on 14 April 2011

We conducted an unmatched retrospective case-control study to investigate an upsurge of non-travelrelated sporadic cases of infection with Salmonella enterica subsp. enterica serotype Enteritidis phage type $14 \mathrm{~b}$ with antimicrobial resistance to nalidixic acid and partial resistance to ciprofloxacin (S. Enteritidis PT $14 \mathrm{~b} \mathrm{Nx}\left(\mathrm{p}_{1}\right)$ that was reported in England from 1 September to 31 December 2009. We analysed data from 63 cases and 108 controls to determine whether cases had the same sources of infection as those found through investigation of 16 concurrent local foodborne outbreaks in England and Wales. Multivariable logistic regression analysis adjusting for age and sex identified food consumption at restaurants serving Chinese or Thai cuisine (odds ratio (OR): $4.4 ; 95 \% \mathrm{Cl}$ : 1.3-14.8; $p=0.02)$, egg consumed away from home (OR: $5.1 ; 95 \% \mathrm{Cl}: 1.3-21.2 ; \mathrm{p}=0.02$ ) and eating vegetarian foods away from home (OR: 14.6; 95\% Cl: 2.1-99; $\mathrm{p}=0.006$ ) as significant risk factors for infection with $S$. Enteritidis PT $14 \mathrm{~b} \mathrm{NxCp_{L }}$. These findings concurred with those from the investigation of the16 outbreaks, which identified the same Salmonella strain in eggs from a specified source outside the United Kingdom. The findings led to a prohibition of imports from this source, in order to control the outbreak.

\section{Introduction}

Infection with Salmonella enterica subsp. enterica serotype Enteritidis (S. Enteritidis) remains an important public health problem in Europe and other parts of the world [1-4]. Outbreaks caused by Salmonella infection have been associated with a variety of foods; however, outbreaks caused by Salmonella Enteritidis infection are closely associated with eggs and egg products $[2,5,6]$. In September 2009, the Department of Gastrointestinal, Emerging and Zoonotic Infections at the Health Protection Agency (HPA) reported a marked upsurge in the number of non-travel-related human cases of infection with $S$. Enteritidis phage type (PT) $14 \mathrm{~b}$ with resistance to nalidixic acid and partial

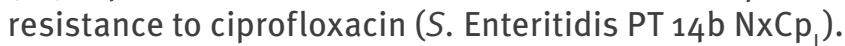
Infectious diseases resulting from food poisoning are statutorily notifiable in England and Wales: cases are notified by registered medical practitioners and diagnostic laboratories to the HPA. In total, 572 cases of

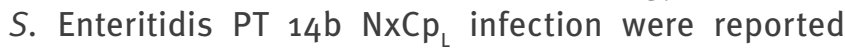
between January and December 2009, compared with 141 in 2008. Between 1 September and 31 December 2009, there were 489 cases.

There were 14 recognised, discrete local outbreaks of S. Enteritidis PT $14 \mathrm{~b} N x \mathrm{p}_{\mathrm{L}}$ infection in England and two in Wales between August and December 2009 (HPA unpublished data). All but one of these outbreaks were linked to food-service premises; the remaining outbreak was linked to a residential care home for the elderly. The total number of reported cases associated with these outbreaks was 152: six were hospitalised and two deaths were reported.

Preliminary investigations of these 16 outbreaks suggested putative links to infected eggs, with evidence

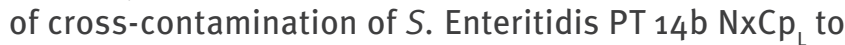
other foods, particularly ready-to-eat vegetarian foods. The outbreak strain was isolated from samples of eggs, egg mayonnaise, egg-fried rice, pooled liquid egg mix and work surfaces in the food-service premises investigated as part of the outbreak investigations. Eggs collected from these premises (five restaurants serving Chinese or Thai cuisine and two cafes) in seven of the outbreaks were from the same production establishment in Spain, as indicated by the stamp on the egg shells. We therefore conducted a case-control analysis to determine whether the likely source of infection in the apparently sporadic cases was the same as that for cases in the outbreaks.

Before the upsurge in $S$. Enteritidis PT $14 \mathrm{~b} N x \mathrm{Cp}_{\mathrm{L}}$ infections in September 2009, there had been other sustained increases in the incidence of $S$. Enteritidis non-PT4 infections in England and Wales between 2000 and 2004 [7,8]. Epidemiological and microbiological investigations and a case-control study of primary sporadic indigenous cases found that consumption of eggs from food prepared outside the home was associated with being a case. The investigations identified 
eggs sourced from Spain used in the food-service sector as the main cause of the increase $[1,2,7,8]$. In the United Kingdom (UK), the predominant PT responsible for egg-borne $S$. Enteritidis infection had been PT 4 between 1992 and 2002 [5]. Following large epidemics of $S$. Enteritidis infection in the UK in the late 1980 s, mainly due to PT 4, a decline in human S .Enteritidis PT 4 infection in England and Wales occurred from 1997, largely because of industry control programmes in the poultry sector, including vaccination of layer flocks [9]. Since 2000, egg-associated S. Enteritidis PTs other than PT 4 causing human infection have emerged, with the greatest increases occurring in S. Enteritidis PT 1and PT 14b-related infections [7].

Surveillance of salmonellosis from 1998 to 2003 also showed upsurges in S. Enteritidis non-PT4 infections in other European countries [1]. Between 1998 and 2003, the proportion of PT4 infections fell from $61.8 \%$ in 1998 to $32.1 \%$ in 2003 , with a concomitant increase in S. Enteritidis non-PT4 infections (including PT1, 8, 14b and 21) in Austria, Germany, Spain, Denmark, Finland, England, Wales and Northern Ireland, Scotland, the Netherlands and Sweden [1]. Major upsurges are thought to be associated with substantive changes in market supply: during this time, eggs were imported from producers in EU Member States where there was a lack of vaccination of layer flocks against Salmonella or controlled food industry assurance schemes were not in place $[1,10,11]$. From 2000 to 2008 , the mean incidence rate for $S$. Enteritidis PT $14 \mathrm{~b} \mathrm{NxCp}$ gradually increased from 0.01 per 100,000 population in England to 0.4 per 100,000 population, respectively. In 2009 , this rate more than doubled, to 1.1 per 100,000 population (HPA unpublished data).

This evidence, along with the findings of the 16 foodborne outbreaks, was used to formulate a hypothesis that $S$. Enteritidis PT $14 \mathrm{~b} \mathrm{NxCp_{L }}$ infection of cases who were not part of the outbreaks was associated with consumption of eggs outside the home, within five

\section{TABLE 1}

Demographic characteristics of sporadic cases of Salmonella Enteritidis PT $14 \mathrm{~b} \mathrm{NxCp}_{\mathrm{L}}$ infection $(\mathrm{n}=63)$ and controls ( $\mathrm{n}=108)$, England, October-December 2009

\begin{tabular}{|l|c|c|}
\hline Characteristic & Number of cases & Number of controls \\
\hline Sex & 28 & 72 \\
\hline Female & 35 & 36 \\
\hline Male & 9 & 2 \\
\hline Age group (years) & 19 & 8 \\
\hline$<10$ & 14 & 38 \\
\hline $10-29$ & 11 & 38 \\
\hline $30-49$ & 10 & 22 \\
\hline $50-69$ & $\mathbf{6 3}$ & $\mathbf{1 0 8}$ \\
\hline$\geq 70$ &
\end{tabular}

$\mathrm{NxCp_{1 }}$ : resistance to nalidixic acid and concomitant reduced susceptibility to ciprofloxacin; PT: phage type. days before symptom onset, particularly at restaurants serving Chinese or Thai cuisine.

\section{Methods}

A unmatched case-control study was carried out to analyse the apparently sporadic cases, recruiting two controls per case, to determine associations between potential risk exposures and symptomatic infection with S. Enteritidis PT $14 \mathrm{~b} \mathrm{NxCp}$. Cases from the 16 food-borne outbreaks were excluded from our study.

Sample size calculations indicated that having data for 60 cases and 120 controls would enable us to detect an odds ratio of 3 (for $50 \%$ of the controls exposed) to 4 (for $10 \%$ of the controls exposed) as being significant at the $5 \%$ level with around $90 \%$ power.

\section{Case definition}

A case of $S$. Enteritidis PT $14 \mathrm{~b} \mathrm{NxCp}_{\mathrm{L}}$ infection was defined as a person in England with abdominal symptoms (diarrhoea and/or vomiting), with an isolate from their stool sample positive for S. Enteritidis PT 14b with resistance to nalidixic acid and concomitant reduced susceptibility to ciprofloxacin, and the isolate received by the HPA Laboratory of Gastrointestinal Pathogens between 1 September and 31 December 2009.

\section{Recruitment and investigation of cases}

Recruitment of cases for the study took place between 1 October and 31 December 2009. Before the data collection period, 12 cases reported in September 2009 were reviewed using local authority food-poisoning questionnaires ('trawling' questionnaires) to assist in generating hypotheses for the possible source of infection. All cases interviewed with this questionnaire were excluded from the study. Cases associated with the 16 discrete food-borne outbreaks were also excluded from this study, as were cases who had travelled outside the United Kingdom within five days of symptom onset and cases who were contacts of other reported cases.

Standardised data were collected on all patients infected with Salmonella (i.e. before the serotype/subtype was known), so that cases and outbreaks could be identified and investigated rapidly. This involved the completion of a standardised questionnaire for each person with presumptive $S$. Enteritidis or laboratoryconfirmed Salmonella infection (all serotypes) by the Health Protection Unit or local authority. The extensive questionnaire included captured data on basic demographics, occupation, details of gastrointestinal illness and any other symptoms, history of travel, and details of food consumption and contact with animals within the five days before symptom onset. Questions on food consumption gathered details of the type and brand of each food consumed, place of purchase, whether the food was consumed in or away from the home, and type of food-service premises visited. The completed questionnaires were sent to the HPA Department of Gastrointestinal, Emerging and Zoonotic Infections for data entry, validation and analysis. Isolates were sent 
to the Salmonella Reference Unit at the HPA Centre for Infections for further characterisation and antimicrobial susceptibility testing $[12,13]$.

\section{Recruitment and investigation of controls}

We used cases' landline telephone numbers, which reflect the location of their domicile, as the basis of the selection of controls (cases who had been contacted by mobile telephone were asked for a landline number). For each case, two controls were recruited using random digit dialling [14]. Controls were therefore chosen from the same telephone exchange area and therefore lived in the same geographical area as the cases. Between 2 October and 2 December 2009, controls were recruited by telephone over five weekday evenings. The individual who picked up the telephone and who agreed to be interviewed was considered to be a control provided they were over the age of 18 years and they provided informed consent on the telephone before the interview.

All interviews were carried out using a standardised questionnaire for controls. This was similar to that used for cases, except that questions on contact with animals, travel history, food consumption and groceryshopping habits related to the five days before the interview (rather than before symptom onset). Controls who had experienced any gastrointestinal symptoms in the two weeks before the interview were excluded from the study.

\section{Data analysis}

The data were analysed using STATA 11. For all exposures, estimated odds ratios and $95 \%$ confidence

\section{TABLE 2}

Single variable analysis of exposure variables for cases of Salmonella Enteritidis PT 14b NxCp $\mathrm{p}_{\mathrm{L}}$ infection ( $\left.\mathrm{n}=63\right)$ and controls $(n=108)$, adjusted for age and sex, England, October-December 2009

\begin{tabular}{|c|c|c|}
\hline Exposure $^{a}$ & Odds ratio $(95 \% \mathrm{Cl})$ & P value \\
\hline \multicolumn{3}{|l|}{ Eaten away from home } \\
\hline Eaten away from home at any type of establishment & $2.6(1.1-5.9)$ & 0.02 \\
\hline Eaten out at parties & $1.5(0.6-3.8)$ & 0.4 \\
\hline \multicolumn{3}{|l|}{ Eaten foods from food-service premises } \\
\hline Restaurants serving Chinese or Thai cuisine & $4.1(1.6-10.4)$ & 0.002 \\
\hline Kebab houses & $17.1(1.7-172)$ & 0.02 \\
\hline Restaurants serving Indian cuisine & $2.7(0.7-9.5)$ & 0.1 \\
\hline Burger bars & $0.5(0.2-1.7)$ & 0.3 \\
\hline Fried chicken bars & $2.2(0.3-17.4)$ & 0.4 \\
\hline Public houses & $0.6(0.2-2.2)$ & 0.5 \\
\hline Restaurants serving Italian cuisine & $1.5(0.4-5.0)$ & 0.5 \\
\hline \multicolumn{3}{|l|}{ Food exposure } \\
\hline Barbecued food & $13.6(1.4-129)$ & 0.02 \\
\hline Eaten barbecued food at home & $9.2(0.9-93)$ & 0.06 \\
\hline Eaten barbecued food away from home & ND & 0.07 \\
\hline Pre-prepared sandwiches & $2.5(1.2-5.4)$ & 0.02 \\
\hline Eaten pre-prepared sandwiches at home & $1.5(0.4-4.9)$ & 0.5 \\
\hline Eaten pre-prepared sandwiches away from home & $3.0(1.3-7.2)$ & 0.01 \\
\hline Vegetarian food & $3.4(1.3-9.2)$ & 0.01 \\
\hline Eaten vegetarian food at home & $1.7(0.6-4.7)$ & 0.3 \\
\hline Eaten vegetarian food away from home & $13.6(2.3-81)$ & 0.004 \\
\hline Cold meats & $1.9(0.9-4.0)$ & 0.08 \\
\hline Eaten cold meats at home & $1.3(0.6-2.5)$ & 0.5 \\
\hline Eaten cold meats away from the home & $8.0(1.7-37)$ & 0.008 \\
\hline Eggs & $1.6(0.7-3.6)$ & 0.3 \\
\hline Eaten eggs eaten at home & $1.00 .5-2.1)$ & 0.97 \\
\hline Eaten eggs eaten away from the home & $7.02 .0-24.8)$ & 0.003 \\
\hline \multicolumn{3}{|l|}{ Environmental exposure } \\
\hline Had contact with animals ${ }^{b}$ & $1.0(0.5-2.0)$ & 0.98 \\
\hline Lived on a farm or smallholding & $4.4(0.2-84)$ & 0.3 \\
\hline Visited a farm & $2.4(0.5-11.5)$ & 0.3 \\
\hline
\end{tabular}

ND: not determined; $N x \mathrm{Cp}_{\mathrm{L}}$ : resistance to nalidixic acid and concomitant reduced susceptibility to ciprofloxacin; PT: phage type.

a The reference category for each exposure is having not eaten at the specified establishment or having not eaten the specified food, or having had the relevant environmental exposure.

${ }^{\mathrm{b}}$ Occupational contact or contact with pets. 
intervals were used as measures of association. In addition, all exposures were tested, singly, for association with the outcome variable (illness) using chisquare test or Fisher's exact test. Exposures exhibiting some evidence of an association ( $p<0.2)$ were deemed eligible for inclusion in the multivariable analysis. The p<0.2 cut-off was chosen so that important exposures would not be missed due to confounding effects. A logistic regression model was constructed using a forward selection procedure including the most significant exposure at each step (likelihood ratio test $\mathrm{p} \leq 0.05)$. Potential confounding variables - age and sex - were included in the multivariable analysis regardless of statistical significance.

\section{Results}

A total of 489 S. Enteritidis PT $14 \mathrm{~b} \mathrm{NxCp}$ cases distributed across all regions of England were identified by the HPA Laboratory of Gastrointestinal Pathogens during the study period. Of these, 101 were associated with the discrete food-borne outbreaks and were therefore excluded. Some cases not associated with these discrete outbreaks were also not included because they were interviewed with the initial trawling questionnaire in September, before the investigation, and others were excluded because they were identified after our investigation had closed. In total,

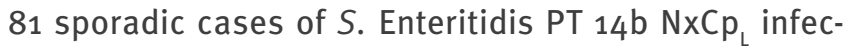
tion completed the questionnaire. Of these 81 cases, 63 were included in the analysis: four were excluded due to recent travel history and 14 were excluded because they were contacts of other cases (although the index cases were included). There were reports of people with $S$. Enteritidis $\mathrm{PT} 14 \mathrm{~b} \mathrm{NxCp_{L }}$ infection after December 2009, but the number reported had fallen to background levels.

A total of 108 controls were recruited: a mean of 3.6 calls (range: 1-32 calls) was needed to successfully recruit a control. Table 1 compares the basic demographic characteristics of cases and controls. Controls were more likely to be female $(p=0.004)$ and older (mean age: 52.5 versus 36.8 years, respectively, compared with cases, p 0.0001$)$. Due to these differences between cases and controls, single variable analysis was performed using logistic regression analysis adjusting for potential confounding by age and sex.
The cases had dates of symptom onset between 26 August and 16 November 2009, and the mean duration of illness in those who had recovered was 7 days (median: 7 days; lower and upper quartiles: 3 and 10 days, respectively). The predominant symptoms were diarrhoea (in 59 of 60 cases), abdominal pain (49 of 56), fever, defined as body temperature of at least 38 ${ }^{\circ} \mathrm{C}$ (32 of 55), nausea (29 of 55), headaches (26 of 55) and vomiting (20 of 59 ). Of the 63 cases, 15 reported having blood in their stool. A total of 50 visited their general practitioner, while 13 attended hospital accident and emergency departments and 12 were admitted to hospital. No deaths were reported among the study cases.

As there could be a delay in reporting (i.e. date of symptom onset was not necessarily the date the cases were reported) and to allow time for isolates to be sent for typing, the cut-off date for receipt of isolates at the HPA Laboratory of Gastrointestinal Pathogens was 31 December 2009.

In single variable analysis there was an association between having eaten away from home and symptomatic infection with $S$. Enteritidis PT $14 \mathrm{~b} \mathrm{NxCp}$, particularly in restaurants serving Chinese or Thai cuisine and kebab houses (Table 2). Having eaten barbecued foods either at home or away from home, and pre-prepared sandwiches obtained away from home, was also associated with a higher risk of becoming a case. There was a very strong association between having eaten eggs away from home and becoming a case (Table 2 ).

As both eating away from home at any type of establishment and eating foods from restaurants serving Chinese or Thai cuisine were found to be significantly associated with being a case, a three-level factor was generated to determine any association between being a case and (1) not eating out, (2) eating out at restaurants serving Chinese or Thai cuisine, and (3) eating out at other restaurants. The final multivariable logistic regression model including the implicated exposure variables (Table 3) demonstrated no significant association between having eaten away from home but not at restaurants serving Chinese or Thai cuisine and becoming a case. However, having eaten foods from restaurants serving Chinese or Thai cuisine (including takeaways) was significantly associated with becoming a case. Among food exposures, eggs eaten away

\section{TABLE 3}

Multivariable logistic regression model of implicated food exposures, adjusted for age and sex, England, October-December $2009(n=63)$

\begin{tabular}{|c|c|c|c|}
\hline Food exposure & Odds ratio $(95 \% \mathrm{Cl})$ & Pvalue & Number of cases exposed \\
\hline Had not eaten away from home & Reference & - & 15 \\
\hline Eaten away from home but not at a restaurant serving Chinese or Thai cuisine & $1.5(0.5-4.1)$ & 0.5 & 24 \\
\hline Eaten foods from restaurants serving Chinese or Thai cuisine & $4.4(1.3-14.8)$ & 0.02 & 25 \\
\hline Eaten eggs away from home & $5.1(1.2-21.2)$ & 0.02 & 12 \\
\hline Eaten vegetarian food away from home & $14.6(2.1-99)$ & 0.006 & 6 \\
\hline
\end{tabular}


from home and vegetarian foods eaten away from home were also identified as significant risk factors for becoming a case.

\section{Discussion and conclusion}

The case-control study presented here provides evidence of significant associations between eating in restaurants serving Chinese or Thai cuisine and eating eggs and vegetarian food away from home with becoming a case of $S$. Enteritidis $\mathrm{PT}_{14} \mathrm{~b} \mathrm{NxCp}_{\mathrm{L}}$ infection in a large national outbreak in England in 2009. The association between eating vegetarian foods and becoming a case may be related to the fact that vegetarian foods may contain eggs (which could be infected). These findings corroborated evidence obtained from concurrent investigations of 16 local discrete food-borne outbreaks of $S$. Enteritidis $\mathrm{PT}_{14} \mathrm{~b} \mathrm{NxCp}$ infection. Our results indicated that the source of infection for the sporadic cases was likely to be the same as that for cases associated with the outbreaks. Information on eggs collected from food-service premises in seven of the 16 outbreaks indicated a common origin (a single production establishment in Spain). S. Enteritidis PT14 $\mathrm{NxCp}$ obtained from eggs from this establishment, and also from environmental and food samples from the food-service premises were indistinguishable by molecular diagnostic testing from isolates obtained from human cases of $S$. Enteritidis $\mathrm{PT}_{14} \mathrm{NxCp_{L }}$ infection (cases associated with the outbreaks and the sporadic cases). $S$. Enteritidis $\mathrm{PT}_{1} \mathrm{NxCp}$, was additionally detected in eggs produced by this establishment in Spain as part of the outbreak investigations [15] providing further evidence of $S$. Enteritidis contamination within the laying flock.

\section{Control measures}

The United Kingdom Food Standards Agency was informed of the findings both from the case-control study and the 16 outbreak investigations and notified the European Commission and other EU Member States in October 2009 through the Rapid Alert System for Food and Feed (RASFF) of the eggs contaminated with S. Enteritidis PT $14 \mathrm{~b} \mathrm{NxCp}$ and also PT $1 \mathrm{NxCp}$, sourced from an approved establishment in Spain (one of the conditions of approval is compliance with all the relevant legislation set out by the relevant EU Member State competent authority). This led to Spanish authorities investigating and identifying the affected flock. Eggs from this flock were prohibited from entering the fresh table egg market and were sent for heat treatment (as required by EU regulations $[15,16]$, which state that eggs from flocks testing positive for $S$. Enteritidis or $S$. Typhimurium need to be treated in a manner that guarantees the elimination of Salmonella). After this control measure was introduced in early December 2009, the number of cases in England and Wales fell from a mean of 20 confirmed cases per week in November to nine and three per week in December and January 2010, respectively.
A decreasing trend in the notification rate of salmonellosis cases in the EU, particularly those caused by $S$. Enteritidis, has been seen over recent years. This has largely been attributed to the implementation of Salmonella national control programmes in the laying flocks [17]. Nevertheless, most of the reported foodborne outbreaks reported in the EU are still caused by Salmonella, with the most important food source being eggs and egg products [17]. Eggs have continued to be implicated as a source of or vehicle for crosscontamination in outbreaks of salmonellosis chiefly associated with the food-service industry in the UK [5-8]. Food-poisoning risks associated with eggs and egg dishes in the food-service industry, especially those serving Chinese cuisine, have included highrisk practices such as breaking, pooling and mixing shelled eggs $[18,19]$. One Salmonella-contaminated egg is capable of contaminating the whole batch of raw shell egg mix, and large numbers of consumers may be exposed to this contaminated raw material. The risk is increased if the egg mix is stored in a warm kitchen for later use during the day, as this would allow growth of the pathogen. Cross-contamination through egg mix aerosolisation during whisking and transfer to utensils and food preparation areas is also of concern [19]. The rates of Salmonella contamination have been linked to the origin of the eggs [20]. The food-service sector and consumers still need to be aware of this continuing hazard and adopt appropriate control measures and follow advice provided by national food safety agencies, in order to reduce the risk of infection.

\section{Study limitations}

Our case-control study had a number of limitations. Firstly, because of the limited time and resources available to recruit the controls, the final number of controls was slightly below the required number, based on our sample-size calculation (108 recruited as opposed to 120). In some of our analyses, small numbers led to large confidence intervals

Secondly, for the recruitment of controls we interviewed the person who answered the telephone (provided they were aged over 18 years), which may have introduced further bias, as we found that those who were most likely to answer were more likely to be older and also female. We did not use a method such as the 'last birthday' method (in which the adult in the household with the most recent birthday is requested for interview during the telephone call) - such an approach might help to increase variation in the demographics of the controls. However, we took measures to try to minimise response bias by varying the days of the week and the times that controls were telephoned. To minimise any potential confounding by age and sex, these were adjusted for in the regression analysis.

Thirdly, recall bias was a potential problem, particularly for controls. When cases were interviewed, they were asked about their food consumption in the five days before becoming ill whereas controls were asked 
about their food consumption in the five days before the telephone interview.

Fourthly, the time period for recruitment of cases did not exactly mirror that for the recruitment of controls, as we recruited controls over five weekday evenings in October and December 2009, whereas cases were recruited over a continuous period throughout October and December 2009.

Finally, we recognise that there may have been further confounders relating to differences in occupation, socio-economic status and eating behaviours between cases and controls. We attempted to minimise these potential confounders by interviewing controls who were living in the same telephone exchange area as cases. We also note that cases were not over-representative of Chinese or Thai ethnic groups (data not shown), so this form of confounding is not relevant to our investigation.

Despite the limitations - most of which are common to case-control studies of outbreak investigations of gastrointestinal infection - the results of the study support our hypothesis.

\section{References}

1. Fisher IS; Enter-net participants. Dramatic shift in the epidemiology of Salmonella enterica serotype Enteritidis phage types in western Europe, 1998-2003: results from the Enter-net international salmonella database. Euro Surveill. 2004;9(11):pii=486. Available from: http://www. eurosurveillance.org/ViewArticle.aspx?Articleld $=486$

2. Centers for Disease Control and Prevention (CDC). Investigation update: multistate outbreak of human Salmonella Enteritidis infections associated with shell eggs. 2 Dec 2010. Available from: http://www.cdc.gov/salmonella/enteritidis/

3. Braden CR. Salmonella enterica serotype Enteritidis and eggs: a national epidemic in the United States. Clin Infect Dis. 2006;43:512-7.

4. Galanis E, Lo Fo Wong DM, Patrick ME, Binsztein N, Cieslik A, Chalermchikit T, et al. Web-based surveillance and global Salmonella distribution, 2000-2002. Emerg Infect Dis. 2006;12(3):381-8

5. Gillespie IA., O’Brien SI, Adak GK, Ward LR, Smith HR. Foodborne general outbreaks of Salmonella Enteritidis phage type 4 infection, England and Wales, 1992-2002: where are the risks? Epidemiol. Infect. 2005;133(5):795-801.

6. Ejidokun 00, Killalea D, Cooper M, Holmyard S, Cross A, Kemp C. Four linked outbreaks of Salmonella enteritidis phage type 4 infection--the continuing egg threat. Commun Dis Public Health. 2000;3(2):95-100.

7. Gillespie I, Elson R. Successful reduction of human Salmonella Enteritidis infection in England and Wales. Euro Surveill. 2005;10(46): pii=2834. Available from: http://www. eurosurveillance.org/ViewArticle.aspx?Articleld =2834

8. Gillespie I. Salmonella Enteritidis non-phage type 4 infections in England and Wales 2000-2004: Report from a multi-agency national outbreak control team. Euro Surveill. 2004;8(42):pii=2569. Available from: http://www. eurosurveillance.org/ViewArticle.aspx?Articleld=2569

9. Cogan TA, Humphrey TJ. The rise and fall of Salmonella Enteritidis in the UK. J Appl Microbiol. 2003;94 Suppl:114S-119S.

10. Nygård K. 12 December 2002. Salmonella Enteritidis in eggs imported to Norway. Euro Surveill, 2002;6(50):pii=1986. Available from: http://www.eurosurveillance.org/ViewArticle. aspx?Articleld $=1986$
11. van Pelt W, Mevius D, Stoelhorst HG, Kovats S, van de Giessen AW, W. Wannet, and Y. T. Duynhoven. 2004. A large increase of Salmonella infections in 2003 in The Netherlands: hot summer or side effect of the avian influenza outbreak? Euro Surveill. 2004;9(7):pii=473. Available from: http://www. eurosurveillance.org/ViewArticle.aspx?Articleld $=473$

12. Frost JA.. Testing for resistance to antimicrobial drugs. In: Chart $\mathrm{H}$, editor. Methods in practical laboratory bacteriology. New York: CRC Press; 1994. p. 73-82.

13. Ward LR, de Sa JD, Rowe B. A phage-typing scheme for Salmonella Enteritidis. Epidemiol Infect. 99(2):291-4.

14. Hartge P, Brinton LA, Rosenthal JF, Cahill Jl, Hoover RN, Waksberg J. Random digit dialing in selecting a populationbased control group. Am J Epidemiol.1984;120(6):825-33.

15. Health Protection Agency (HPA). National increase in Salmonella Enteritidis phage type $14 \mathrm{~b} \mathrm{NxCpl}$ infections in England - an update. Health Protection Report 3(47); 27 November 2009. Available from: http://www.hpa.org.uk/hpr/ archives/2009/news4709.htm

16. European Commission. Commission Regulation 1237/2007 of 23 October 2007 amending Regulation 2160/2003 of the European Parliament and of the Council, and Decision $2006 / 696 / E C$, as regards placing on the market of eggs from Salmonella infected flocks of laying hens. Official Journal of the European Union, L 280, 5-9. Available from: http://eurlex. europa.eu/LexUriServ/LexUriServ.do?uri=0J:L:2007:280:0005 :0009:EN:PDF

17. European Food Safety Authority (EFSA). The Community summary report on trends and sources of zoonoses, zoonotic agents and food-borne outbreaks in the European Union in 2008. EFSA Journal. 2010,8(1):1496.

18. Little CL, Rhoades JR, Hucklesby L, Greenwood M, Surman-Lee $\mathrm{S}$, Bolton FJ, et al. Survey of Salmonella contamination of raw shell eggs used in catering premises in the United Kingdom, 2005 through 2006. J Food Prot. 71(1);19-26.

19. Gormley FJ, Little CL, Murphy N, de Pinna E and McLauchlin J. Pooling raw shell eggs: Salmonella contamination and high risk practices in the United Kingdom food service sector. J Food Prot. 73(3); 574-8.

20. European Food Safety Authority (EFSA). Report of the Task Force on Zoonoses Data Collection on the analysis of the baseline study on the prevalence of Salmonella in holdings of laying hen flocks of Gallus gallus. EFSA Journal. 2007;97. 